\title{
EXPERIENCIA DE ENSINO E APRENDIZAGEM DE FARMACOLOGIA EM MEDICINA VETERINÁRIA, EM ALEGRE- ES
}

\author{
Rudison da Silva Florêncio' \\ Mirelle Baptista Jordaim ${ }^{2}$ \\ Débora de Souza Pozzatto ${ }^{3}$ \\ Amanda Maria Miranda Rodrigues dos Santos ${ }^{4}$ \\ Rosilene do Carmo Mendes ${ }^{5}$ \\ Thaís Portes Deorce Pimenta ${ }^{6}$ \\ Gabriela Fiuza Corato ${ }^{7}$ \\ Caio Vaz Baqui Lima ${ }^{8}$ \\ Mariana Corrêa Santos ${ }^{9}$ \\ Lenir Cardoso Porfírio ${ }^{10}$
}

Resumo: Em medicina veterinária a farmacologia é uma das disciplinas que une o ciclo básico com o ciclo profissionalizante, sendo constantemente utilizada na clínica médica e suas disciplinas relacionadas, além de possuir importante papel em áreas não relacionadas com clínica, cirurgia e áreas relacionadas. $O$ estudo de farmacologia auxilia na formação de profissionais médicos veterinários, habilitando-os a utilizar os diferentes medicamentos e o conhecimento sobre eles para diagnosticar, tratar e prevenir doenças dos animais, e assim, reduzir seu sofrimento. Este trabalho mostra resultados de um modo de ensino e aprendizagem na disciplina de farmacologia básica do curso de medicina veterinária da Universidade Federal do Espírito Santo, Alegre, ES. Foram utilizados recursos de monitoria, estudos de casos clínicos, aliados a seminários e resolução de estudos dirigidos, durante o período de março a julho de 2016. Concluise que é possivel melhorar assimilação de conteúdo por meio da combinação de estratégias de ensino e aprendizagem, com apresentação de casos clínicos e monitorias com grupos pequenos de estudantes.

Palavras-chave: Estratégias de Ensino; Terapêutica Veterinária; Farmacologia Clínica.

\footnotetext{
${ }^{1}$ Medicina Veterinária/Universidade Federal do Espirito Santo Brasil. E-mail: rudi.florencio@gmail.com.

2 Medicina Veterinária/Universidade Federal do Espirito Santo Brasil. E-mail: mirelle_bj@gmail.com.

${ }^{3}$ Medicina Veterinária/Universidade Federal do Espirito Santo Brasil. E-mail: pozzato.debora@hotmail.com.

${ }^{4}$ Medicina Veterinária/Universidade Federal do Espirito Santo Brasil. E-mail: amanda_mmrs@hotmail.com.

${ }^{5}$ Medicina Veterinária/Universidade Federal do Espirito Santo Brasil. E-mail: rosilenemendess@hotmail.com.

${ }^{6}$ Medicina Veterinária/Universidade Federal do Espirito Santo Brasil. E-mail: thaispimeenta@gmail.com.

7 Medicina Veterinária/Universidade Federal do Espirito Santo Brasil. E-mail: gabrielafiuza@hotmail.com.

${ }^{8}$ Medicina Veterinária/Universidade Federal do Espirito Santo Brasil. E-mail: caiobaqui@hotmail.com.

${ }^{9}$ Medicina Veterinária/Universidade Federal do Espirito Santo Brasil. E-mail: mariana.correasantos@gmail.com.

${ }^{10}$ Medicina Veterinária/Universidade Federal do Espirito Santo Brasil. E-mail: lenircp52@gmail.com.
} 